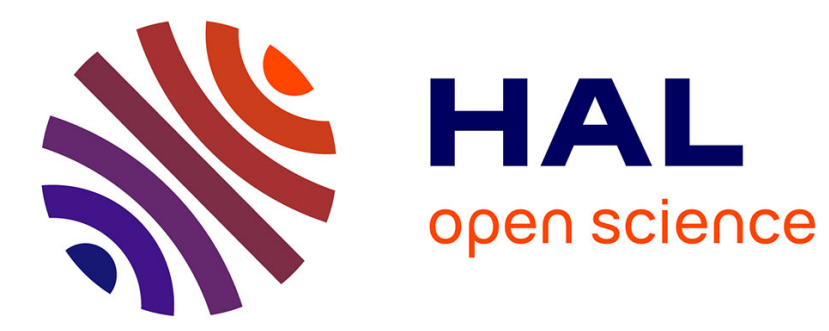

\title{
La propriété immobilière est-elle un obstacle pour sortir du chômage?
}

Carole Brunet, Nathalie Havet, Jean-Yves Lesueur

\section{To cite this version:}

Carole Brunet, Nathalie Havet, Jean-Yves Lesueur. La propriété immobilière est-elle un obstacle pour sortir du chômage?. 2010. halshs-00477236

\section{HAL Id: halshs-00477236 \\ https://shs.hal.science/halshs-00477236}

Submitted on 28 Apr 2010

HAL is a multi-disciplinary open access archive for the deposit and dissemination of scientific research documents, whether they are published or not. The documents may come from teaching and research institutions in France or abroad, or from public or private research centers.
L'archive ouverte pluridisciplinaire HAL, est destinée au dépôt et à la diffusion de documents scientifiques de niveau recherche, publiés ou non, émanant des établissements d'enseignement et de recherche français ou étrangers, des laboratoires publics ou privés. 
La propriété immobilière est-elle un obstacle pour sortir du chômage?

Carole Brunet, Nathalie Havet, Jean-Yves Lesueur

Avril 2010 


\section{GATE Groupe d'Analyse et de Théorie Économique Lyon-St Étienne}

93, chemin des Mouilles 69130 Ecully - France

Tel. +33(0)4 72866060

Fax $+33(0) 472866090$

6, rue Basse des Rives 42023 Saint-Etienne cedex 02 - France

Tel. +33 (0)4 77421960

Fax. $+33(0) 477421950$

Messagerie électronique / Email : gate@gate.cnrs.fr

Téléchargement / Download : http://www.gate.cnrs.fr - Publications / Working Papers 


\title{
La propriété immobilière est-elle un obstacle pour sortir du chômage ?*
}

\author{
Carole Brunet ${ }^{\dagger}$ \\ LED \\ Université Paris 8
}

\author{
Nathalie Havet $\ddagger$ \\ GATE Lyon-Saint Etienne \\ Université de Lyon, CNRS.
}

\author{
Jean-Yves Lesueur $\S$ \\ GATE Lyon-Saint Etienne \\ Université de Lyon, CNRS.
}

\begin{abstract}
Résumé
Cet article propose de tester, sur données individuelles, l'hypothèse d'Oswald, selon laquelle le développement de la propriété immobilière observé dans la plupart des pays de l'OCDE s'accompagnerait d'une augmentation de la durée des épisodes de chômage. La démarche retenue apporte une contribution à la littérature dans ce domaine via une étude économétrique comparée entre la France et les Etats-Unis qui permet d'opposer des fonctionnements et des interactions entre marchés du travail et du logement particulièrement différenciés. A partir de deux échantillons de chômeurs issus respectivement du Panel Européen des Ménages et du Panel Survey of Income Dynamics et couvrant la période 1994-2001, des modèles de hasards proportionnels mélangés tenant compte de la simultanéité des comportements sur le marché du travail et celui du logement sont estimés. Les principaux résultats indiquent que la propriété immobilière n'a pas d'impact statistiquement significatif sur les durées de chômage aux Etats-Unis, tandis que les propriétaires français au chômage retrouvent un emploi plus rapidement que leurs homologues locataires, résultats qui conduisent à moduler les conclusions issues de travaux antérieurs appliqués à ces deux pays.
\end{abstract}

Mots clés : Statut résidentiel, durée de chômage, hypothèse d'Oswald

\begin{abstract}
Does Homeownership hinder the exits from unemployment? : This study uses microeconomic data in order to test the so-called Oswald's hypothesis, according to which rising homeownership rates observed in most OECD countries relate to longer unemployment spells. We contribute to the existing literature by contrasting econometric results for
\end{abstract}

\footnotetext{
${ }^{*}$ Les auteurs tiennent à remercier la DARES pour son soutien financier. Ils remercient aussi Alexis Penot ainsi que les membres du comité de suivi du projet «Mobilité professionnelle » pour leurs précieuses suggestions et remarques. Les opinions exprimées et les analyses développées dans cet article restent néanmoins propres aux auteurs.

${ }^{\dagger}$ LED, Département d'Economie. 2, rue de la Liberté 93526 SAINT-DENIS CEDEX. E-mail : carole.brunet@univ-paris8.fr

${ }^{\ddagger}$ GATE, 93, chemin des Mouilles B.P. 16769131 ECULLY. E-mail : havet@gate.cnrs.fr

${ }^{\S}$ GATE, 93, chemin des Mouilles B.P. 16769131 ECULLY. E-mail : lesueur@gate.cnrs.fr
} 
France and United States. We thus compare much differentiated functioning and interactions of labour and housing markets. Using unemployment spells samples from the French part of the European Community Household Panel and the Panel Survey of Income Dynamics, both covering years 1994-2001, we estimate mixed proportional hazard models taking into account simultaneous behaviours on the housing and labour markets. Main results indicate that homeownership is not significantly related to longer unemployment durations in the US, while French homeowners exhibit faster re-employment rates than renters. These findings motivate a re-examination of previous studies conclusions.

Keywords : residential status, unemployment duration, Oswald's hypothesis.

JEL : C41, J64, R21. 


\section{Introduction}

Dans la plupart des pays de l'OCDE, les politiques publiques ont été depuis la fin de la deuxième Guerre Mondiale un moteur du développement de la propriété immobilière. La littérature économique récente a essayé d'évaluer les répercussions indirectes potentielles de telles mesures influençant le statut résidentiel sur le fonctionnement du marché du travail. Un éclairage particulier a notamment été porté ces dernières années sur la nature et le sens des liens entre les choix résidentiels et la qualité de l'insertion professionnelle des individus sur le marché du travail. En effet, la situation en matière de logement est susceptible d'influencer la mobilité professionnelle, en conditionnant les offres d'emploi accessibles physiquement à l'individu et en affectant sa mobilité géographique. Plus récemment, la crise des suprimes aux Etats-Unis et sa diffusion à l'économie réelle à l'échelle internationale ont rappelé les liens étroits entre le secteur immobilier et les autres compartiments de l'économie, et posé de façon cruciale la question de la soutenabilité d'une politique économique de promotion de la propriété immobilière déconnectée des aspects économiques réels, notamment ceux relatifs au marché du travail.

L'évaluation de l'impact de la propriété immobilière sur l'incidence et la durée de chômage a été une des questions les plus populaires et les plus controversées dans la littérature traitant des interactions entre les marchés de l'emploi et du logement. Les travaux d'Oswald (1996, 1998, 1999) ont initié ce débat dans les années quatre-vingt-dix, en suggérant que la répartition du mode d'occupation des logements entre propriétaires et locataires était une explication alternative aux déterminants usuels (système d'indemnités chômage, pouvoir des syndicats, rigidité des salaires, taux d'imposition, etc) des différences internationales ou interrégionales entre taux de chômage observés en Europe et aux Etats-Unis. Sur la période 1960-1990, une corrélation statistiquement significative et positive entre taux de chômage et proportion de propriétaires est ainsi manifeste dans les principaux pays de l'OCDE, tant au niveau national qu'entre régions au niveau intra-national. Quoique mise en évidence au niveau macroéconomique, cette relation positive, connue désormais sous le nom « d'hypothèse d'Oswald », est justifiée par des fondements essentiellement microéconomiques.

Oswald (1999) avance plusieurs mécanismes sous-jacents à ce phénomène, basés pour la plupart sur l'argument que la propriété immobilière est un obstacle à la mobilité, pourtant nécessaire à l'efficacité du marché du travail. Les coûts de mobilité élevés associés à la propriété immobilière auraient des répercussions sur trois catégories d'actifs : les propriétaires au chômage, les propriétaires en emploi et les chômeurs y compris locataires. Premièrement, la propriété immobilière restreint le bassin d'emplois envisageables pour une embauche, ce qui diminue l'efficacité de la recherche d'emploi des chômeurs ayant opté pour ce statut résidentiel. Dans cette optique, une augmentation du nombre de propriétaires entraîne une réduction des possibilités d'adéquations entre chercheurs d'emplois et emplois vacants, générant une hausse du taux de chômage. Deuxièmement, les contraintes de mobilité occasionnées par la propriété immobilière jouent négativement sur le processus d'appariement des propriétaires en emploi. Ces derniers sont moins enclins que les locataires à chercher un nouvel emploi et/ou le font 
dans un bassin plus limité, se privant par là-même d'offres d'emplois intéressantes qui auraient amélioré la qualité de leur appariement. La propriété immobilière favorise donc la persistance des mauvaises adéquations salarié-emploi, rendant l'économie moins efficace (coûts de production plus élevés, diminution des revenus réels) ce qui à long terme pourrait être une source de destructions d'emplois. Troisièmement, les marchés du logement caractérisés par une proportion élevée de propriétaires empêchent les chômeurs, et notamment les plus jeunes sans apports financiers, de trouver un logement approprié proche des emplois souhaités. L'imparfaite allocation spatiale des emplois et des chômeurs limite à nouveau les adéquations possibles entre chômeurs et emplois vacants.

Au delà des justifications apportées par Oswald, les travaux développés sur la formation non concurrentielle des salaires en situation de monopsone [Manning (2003)] peuvent également trouver pour fondement une accélération du taux d'accès à la propriété. En effet sur un marché local, si l'augmentation du nombre de propriétaires réduit la mobilité des travailleurs, les firmes peuvent tirer avantage de ces coûts de friction sur ce bassin d'emplois pour pratiquer des salaires en dessous de la référence concurrentielle. Ainsi peuvent apparaître une discrimination salariale à l'égard des propriétaires et/ou des difficultés pour les firmes à pourvoir les postes vacants. Les théories de la mobilité spatiale [Simpson (1992), Buchel \& van Ham (2003)] mettent de plus en avant la substitution existant entre chômage et mauvais appariement pour un travailleur confronté à l'absence d'emploi adapté sur le marché local et à des coûts de mobilité élevés.

Cet article s'inscrit dans la lignée des travaux microéconométriques qui cherchent à tester empiriquement la pertinence du premier mécanisme évoqué et propose une mise en perspective originale des cas français et américain. Plus précisément, on examine si en France et aux Etats-Unis, les propriétaires ont des durées de chômage plus longues que les locataires, toutes choses étant égales par ailleurs, comme le suggère l'hypothèse d'Oswald. La comparaison des deux pays est intéressante en ce sens qu'elle est tout à fait emblématique de fonctionnements des marchés du travail et du logement opposant une régulation plutôt concurrentielle pour les Etats-Unis à une logique plus réglementée pour la France. Pour ce faire, on exploite la partie française du Panel Européen des Ménages 1994-2001 (PEM) et les données américaines du Panel Study of Income Dynamics 1994-2001 (PSID). Les études les plus récentes et les plus robustes, appliquées à d'autres pays, plaident plutôt pour une réfutation de l'hypothèse d'Oswald. Munch, Rosholm \& Svarer (2006) pour le Danemark et van Vuuren (2009) pour les Pays-Bas trouvent ainsi que le fait d'être propriétaire réduit la durée des épisodes de chômage. Battu, Ma \& Phimister (2008) concluent, quant à eux, pour le Royaume-Uni qu'il n'existe pas de différence significative du point de vue des sorties du chômage entre propriétaires et locataires. Des études ont déjà été menées sur ce thème pour les Etats-Unis [Green \& Hendershott (2001)] et pour la France [Brunet \& Lesueur (2009, 2004), Brunet, Clark \& Lesueur (2007)] mais séparément pour les deux pays. Elles montraient plutôt un effet positif de la propriété immobilière sur la durée de chômage. Néanmoins, leurs conclusions méritent d'être confortées par une modélisations économétrique plus sophistiquée et identique pour les deux pays. En effet, le modèle de durée utilisé par Green \& Hendershott (2001) est fondé sur une distribution Weibull et ne tient pas compte de l'hétérogénéité des caractéristiques inobservées. Les estima- 
tions conduites par Brunet \& Lesueur $(2009$, 2004) et Brunet et al. (2007) permettent de traiter cet aspect, mais la méthode retenue présente l'inconvénient d'être complètement paramétrique et donc sensible aux erreurs de spécifications. Par ailleurs, ces quatre études reposent sur l'utilisation de variables instrumentales et sont menées en deux étapes, sans exploiter la possibilité d'estimer conjointement les équations de statut résidentiel et de durée de chômage.

L'intérêt de la comparaison des effets du statut résidentiel pour ces deux pays apparaît à plusieurs niveaux. Premièrement, si la France et les Etats-Unis enregistrent une forte progression du taux de propriétaires depuis le début des années cinquante, l'ampleur du phénomène et son évolution ne témoignent pas de la même dynamique dans ces deux pays. Le graphique 1 montre que le taux de propriétaires aux Etats-Unis était d'environ 63\% en 1965 et est resté relativement stable jusqu'en 1995. Ensuite, il a augmenté très rapidement pour atteindre un pic à $69 \%$ en 2004 avant de diminuer depuis. La proportion de propriétaires est beaucoup plus faible en France, même si l'écart avec les Etats-Unis s'est réduit durant cette période. L'accession à la propriété en France s'est régulièrement accrue passant de près de $43 \%$ en 1965 à $55 \%$ en 1985. Elle s'est stabilisée à la fin des années 80 et durant la première moitié des années 90 avant de connaître une reprise depuis 1997 [Dubujet \& Blanc (2000), Daubresse (2003), Bosvieux (2005)].

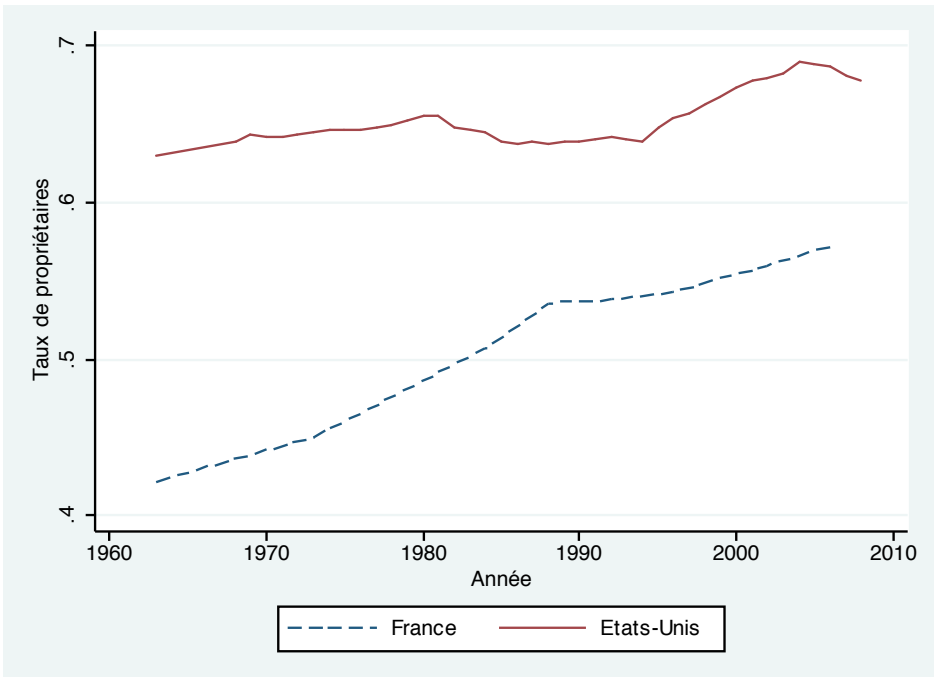

Sources : Enquête Logement pour la France, Census Bureau pour les Etats-Unis

Figure 1 - Evolution du taux de propriétaires en France et aux Etats-Unis

Deuxièmement, au-delà des effets spécifiques attendus dans chaque pays des politiques de logement, des contraintes rencontrées sur le marché du crédit et de la dynamique des prix et des loyers, la structure du marché du travail et son environnement macroéconomique permettent d'envisager des liens très typés entre statut résidentiel et chômage. En comparaison internationale, et en particulier par rapport aux Etats-Unis, le marché du travail français se caractérise 
par un taux de chômage élevé et un chômage de longue durée important, une faiblesse des taux d'activité aux deux classes d'âges extrêmes, un degré d'utilisation des ressources en main d'œuvre particulièrement faible, des normes de protection des emplois très strictes, générant une moindre flexibilité et un fort dualisme entre emplois précaires et emplois stables, et enfin des inégalités salariales moins élevées (Figure 2).
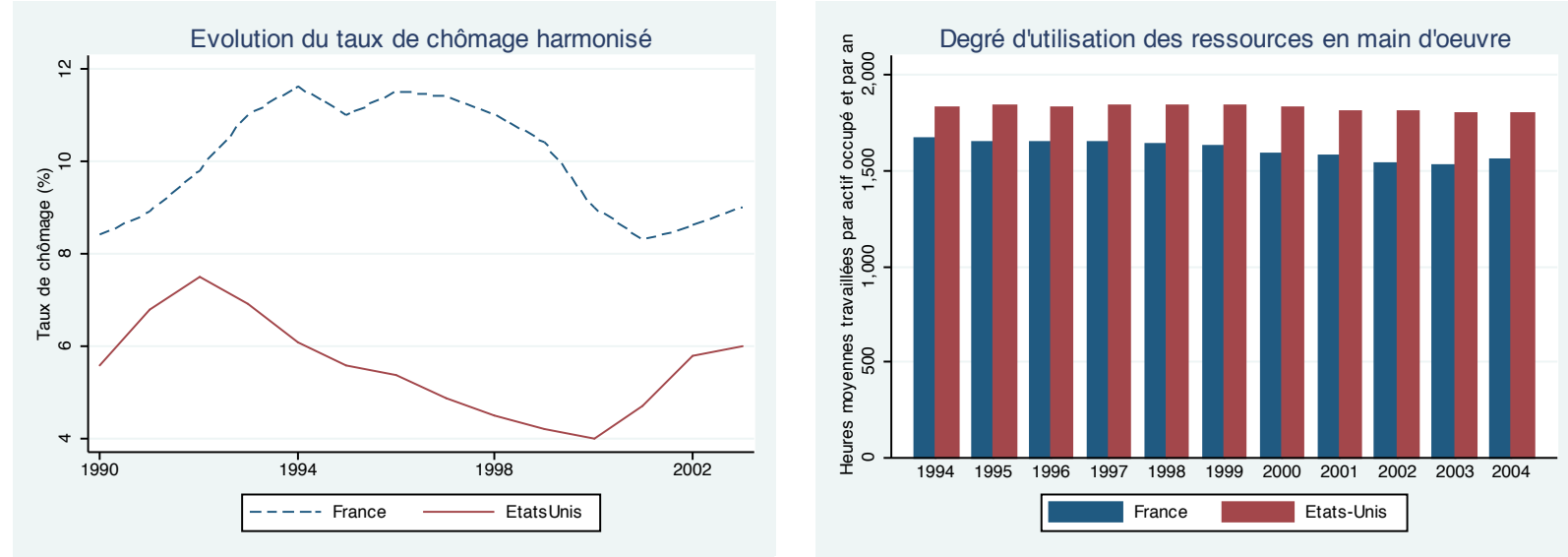

Indice de protection de l'emploi de l'OCDE (1990-2000)

\begin{tabular}{|c|c|c|}
\hline & France & E-U \\
\hline Ens. des contrats & 3,0 & 0,2 \\
Emplois réguliers & 2,4 & 0,2 \\
Emplois temporaires & 3,6 & 0,3 \\
\hline
\end{tabular}

\begin{tabular}{|l|c|c|}
\hline & France & E-U \\
\hline Décile 5 / Décile 1 & 1,53 & 2,05 \\
Décile 9 / Décile 1 & 3,04 & 4,49 \\
Décile 9 / Décile 5 & 1,99 & 2,19 \\
\hline
\end{tabular}

Source : OCDE

FIgURE 2 - Caractéristiques des marchés du travail français et américain

Enfin, la comparaison des effets du statut résidentiel pour ces deux pays est d'autant plus intéressante que les Français et les Américains partagent des sentiments patrimoniaux différents à l'égard de leur logement. Par ailleurs, beaucoup d'éléments indiquent que les restrictions de mobilité associées à la propriété sont bien moindres Outre-Atlantique. En la matière, on peut citer le très important différentiel des frais divers (y compris fiscaux) associés aux transactions immobilières. L'étude de Murray (2007, p.130) montre par exemple que dans le cas d'une maison d'une valeur de 250000 euros, les taxes liées au transfert de propriété s'élèvent à 12725 euros en France contre seulement 837 euros dans l'état du Maine et 761 dans celui de New York. C'est pourquoi, notre comparaison France/Etats-Unis de l'impact du statut résidentiel sur les durées de chômage permettra d'avoir un éclairage sur le rôle des degrés de flexibilité du marché du travail et du marché du logement et sur les éventuelles coordinations à envisager entre les politiques de l'emploi et les politiques du logement. 
Le reste de cet article est organisé comme suit. La section 2 présente les données françaises du Panel Européen des Ménages 1994-2001 (PEM) et les données américaines du Panel Study of Income Dynamics 1994-2001 (PSID). La section 3 détaille la méthode économétrique choisie pour modéliser l'influence de la propriété immobilière sur les durées de chômage. Enfin, les résultats des estimations seront commentés dans la section 4 .

\section{Bases de données mobilisées et analyse descriptive}

\subsection{Constitution des échantillons}

Notre échantillon français a été constitué à partir de la partie française des 8 vagues du Panel Européen des Ménages (PEM) couvrant la période 1994-2001 ${ }^{1}$. Les données mobilisées dans le cas américain sont extraites du Panel Study of Income Dynamics (PSID) ${ }^{2}$, dont on limite l'utilisation aux vagues 1994-2001 pour des raisons de comparabilité avec l'échantillon français.

Ces deux enquêtes ont la particularité d'être des panels d'individus : chacune des personnes du ménage a été interrogée une première fois, puis elles sont suivies d'une année à l'autre même en cas de déménagement ou de changement dans la composition du ménage. Dans les panels de logement, comme l'Enquête Emploi, les logements sont ré-enquêtés que leurs occupants aient changé de domicile ou non. Ainsi, une part importante de l'échantillon correspond à de nouveaux enquêtés et seules les trajectoires d'activité des personnes n'ayant jamais déménagé peuvent être reconstituées. Un des avantages du PEM et du PSID pour notre étude est donc de limiter le biais potentiel lié à l'utilisation d'un sous-échantillon de personnes n'ayant jamais déménagé et d'avoir une meilleure représentativité des différents statuts résidentiels par rapport à l'ensemble de la population.

En outre, ces enquêtes collectent des informations longitudinales sur les éléments de biographie des individus, permettant de reconstituer notamment leur trajectoire sur le marché du travail et sur les conditions de vie du ménage, en particulier celles liées au logement. Elles recueillent auprès des individus des données sur les épisodes de chômage, leur capital humain et autres informations démographiques habituellement disponibles dans les enquêtes auprès des ménages (âge, situation matrimoniale, niveau de scolarité, milieu social, etc). Toutefois, le $P E M$ et le PSID ne contiennent pas de renseignements précis sur le lieu de résidence (hormis le département et la taille de l'unité urbaine pour le PEM et l'Etat pour le PSID). Ainsi, il nous sera impossible d'introduire comme déterminants du choix du statut résidentiel des indices de prix de vente des biens immobiliers, du niveau des loyers dans la commune ou du taux de logements vacants qui auraient pu capter le coût relatif de la location à la propriété. Toutefois les informations disponibles sur la taille des unités urbaines et la dispersion du niveau des taxes

1. Voir Ansieau, Breuil-Genier \& Hourriez (2001) et Breuil-Genier \& Valdelièvre (2001) pour plus de détails.

2. Pour une description détaillée du PSID, voir McGonagle \& Schoeni (2006) ou se reporter à http ://psidonline.isr.umich.edu/. 
locales au lieu de résidence, variables fortement corrélées avec les conditions de prix du marché immobilier et les aménités associées au logement, nous ont permis de contrôler indirectement ces effets inobservés.

Nous restreignons la base de données issue du PEM (respectivement du PSID) aux individus du panel âgés d'au moins dix-sept ans et de moins de 60 ans (respectivement moins de 65 ans), qui vivent dans un ménage de type conventionnel. Nous excluons les ménages constitués de familles impliquant des ascendants indirects ou plus d'un ascendant direct, ainsi que les enfants vivant encore au domicile parental, et en particulier les enfants majeurs considérés comme "logés à titre gratuit". En effet, pour ces derniers, l'éventuelle accession à la propriété correspondrait à une décohabitation parentale qui est motivée par une logique différente des autres décisions de statut résidentiel [Laferrère (2005)] et leurs trajectoires sur le marché du travail relèvent aussi de modes d'insertion spécifique. De même, pour les données françaises, on a exclu les locataires du secteur public dont les parcours sur le marché de l'emploi sont caractérisés par une précarité plus marquée et donc des trajectoires professionnelles qui méritent d'être analysées séparément.

Enfin, à partir des fichiers d'activité du PEM et du PSID, on a reconstruit mois par mois les trajectoires individuelles en définissant trois états sur le marché du travail : en emploi, au chômage et en inactivité. Comme les études récentes [Munch et al. (2006), Barceló (2006), van Vuuren (2009) et Battu et al. (2008)], notre analyse économétrique des durées de chômage se base sur un échantillon constitué uniquement d'entrants au chômage. Sont éliminés de l'échantillon les épisodes de chômage dont la date de commencement est antérieure au début de l'enquête : on s'affranchit ainsi des questions afférentes aux conditions initiales et à des problèmes de censure à gauche des épisodes de chômage. Il reste alors approximativement 3 600 épisodes (pour environ 2000 individus) pour la France et 1571 épisodes pour les EtatsUnis (pour 1369 individus) dont les propriétés sont présentées dans le Tableau 1. Sur nos deux échantillons, la proportion d'occupants propriétaires s'élève à environ $45 \%$ tandis que les locataires représentent 55\%. La prédominance des locataires par rapport aux taux officiels (reportés sur la figure 1) n'est pas surprenante et s'explique par le fait que les propriétaires sont caractérisés par un taux de chômage plus faible. Ainsi, nos échantillons, constitués uniquement de chômeurs, conduisent à une sur-représentation des locataires. Ce phénomène avait déjà été mis en évidence par Munch et al. (2006) dans leur étude sur le Danemark.

\subsection{Caractéristiques des épisodes de chômage}

Sur l'échantillon français, la durée moyenne de chômage est d'environ dix mois, mais les propriétaires se situent au dessus de cette valeur avec une durée moyenne de leurs épisodes de chômage de presque un an, tandis que celle des locataires s'établit à moins de neuf mois. Plus précisément, la moitié des épisodes de chômage des locataires français prend fin avant d'atteindre une durée de six mois tandis que $60 \%$ des épisodes de chômage expérimentés par des individus propriétaires perdurent au-delà. Pour les Etats-Unis, la distribution des durées 


\begin{tabular}{|c|c|c|c|c|c|c|}
\hline & \multicolumn{6}{|c|}{ Statut résidentiel } \\
\hline & 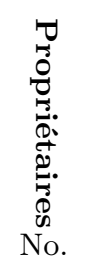 & 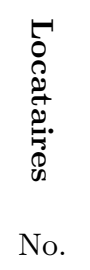 & $\begin{array}{l}\stackrel{1}{0} \\
\stackrel{+}{0}\end{array}$ & 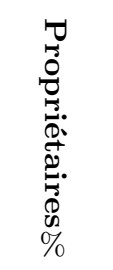 & 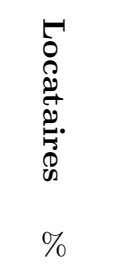 & 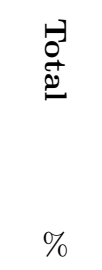 \\
\hline \multicolumn{7}{|l|}{ PEM } \\
\hline \multicolumn{7}{|l|}{ Durée de chômage } \\
\hline Chômage $<1$ mois & 104 & 177 & 281 & 6,41 & 8,67 & 7,67 \\
\hline Chômage $1-2$ mois & 161 & 283 & 444 & 9,92 & 13,87 & 12,12 \\
\hline Chômage 3-5 mois & 410 & 635 & 1045 & 25,26 & 31,11 & 28,52 \\
\hline Chômage 6 -12 mois & 441 & 545 & 986 & 27,17 & 26,70 & 26,91 \\
\hline Chômage 1-2 ans & 295 & 266 & 561 & 18,18 & 13,03 & 15,31 \\
\hline Chômage $>2$ ans & 212 & 135 & 347 & 13,06 & 6,61 & 9,47 \\
\hline \multicolumn{7}{|l|}{ Type de sortie } \\
\hline Censure & 563 & 588 & 1151 & 34,69 & 28,81 & 31,41 \\
\hline Sortie vers l'emploi & 1060 & 1453 & 2513 & 65,31 & 71,19 & 68,59 \\
\hline Total & 1623 & 2041 & 3664 & 100,00 & 100,00 & 100,00 \\
\hline \multicolumn{7}{|l|}{ PSID } \\
\hline \multicolumn{7}{|l|}{ Durée de chômage } \\
\hline Chômage $<1$ mois & 159 & 143 & 302 & 22,05 & 16,82 & 19,22 \\
\hline Chômage 1-2 mois & 33 & 38 & 71 & 4,58 & 4,47 & 4,52 \\
\hline Chômage $3-5$ mois & 260 & 302 & 562 & 36,06 & 35,53 & 35,77 \\
\hline Chômage 6-12 mois & 204 & 287 & 491 & 28,29 & 33,76 & 31,25 \\
\hline Chômage 1-2 ans & 41 & 64 & 105 & 5,69 & 7,53 & 6,68 \\
\hline Chômage $>2$ ans & 24 & 16 & 40 & 3,33 & 1,88 & 2,55 \\
\hline \multicolumn{7}{|l|}{ Type de sortie } \\
\hline Censure & 236 & 269 & 505 & 32,73 & 31,65 & 32,15 \\
\hline Sortie vers l'emploi & 485 & 581 & 1066 & 67,27 & 68,35 & 67,85 \\
\hline Total & 721 & 850 & 1571 & 100,00 & 100,00 & 100,00 \\
\hline
\end{tabular}

TABleau 1 - Caractéristiques des épisodes de chômage

de chômage est quelque peu différente. Le Tableau 1 fait ressortir que les épisodes de chômage ont des durées moyennes plus courtes aux Etats-Unis qu'en France. Aux Etats-Unis, 19\% des épisodes ont une durée inférieure à un mois contre seulement $8 \%$ en France. Par ailleurs, la proportion de propriétaires est beaucoup plus importante que celle des locataires aux deux extrémités de la distribution, c'est-à-dire parmi les épisodes de chômage de très courtes durées (< à 1 mois) et parmi les épisodes de longue durée ( $>$ à 2 ans). Les locataires américains connaissent quant à eux plus fréquemment des épisodes de chômage d'une durée comprise entre six mois et un an. Mais au final, la durée moyenne des épisodes de chômage est quasiment identique entre propriétaires et locataires. 
Les différences de durée de séjour dans l'état de chômage entre les modes d'occupation du logement se répercutent sur les proportions d'épisodes censurés. En particulier, une conséquence de la forte concentration des propriétaires français parmi les épisodes de chômage longs est une plus grande proportion d'épisodes censurés lorsqu'ils correspondent à des individus propriétaires (35\% versus $29 \%$ pour les locataires). Sachant que seules les sorties de chômage relatives à un accès à l'emploi sont considérées ${ }^{3}$, on observe, sur le $P E M$, ce type de transitions pour respectivement $65 \%$ et $71 \%$ des épisodes de chômage des propriétaires et des locataires. En revanche, sur les données du PSID, on observe des taux de censures et de sorties vers l'emploi identiques pour les locataires et les propriétaires variant entre $67 \%$ et $68 \%$.

Les fonctions de survie (estimateur non-paramétrique de Kaplan-Meier) de la figure 3 confirment qu'en France, les propriétaires ont des durées de chômage significativement plus longues que les locataires alors que l'écart entre les deux groupes n'est pas statistiquement significatif aux Etats-Unis ${ }^{4}$.

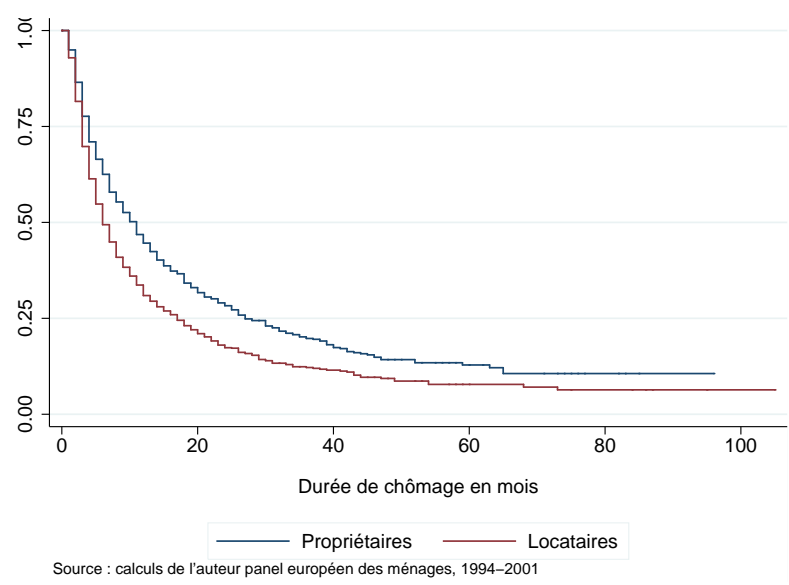

France

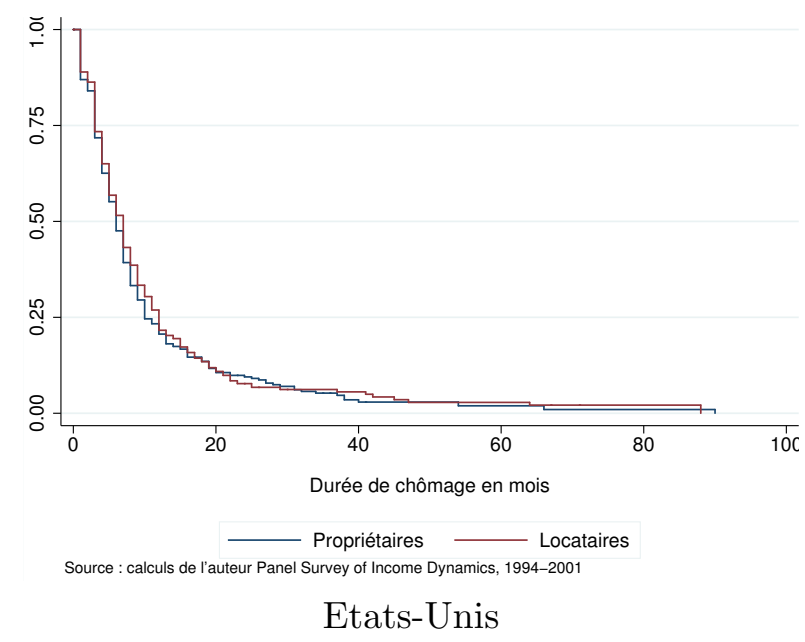

Etats-Unis

FiguRE 3 - Fonctions de survie dans le chômage, Locataires vs. Propriétaires

Toutefois ces résultats descriptifs ne suffisent pas pour conclure que la propriété immobilière est un frein ou un avantage du point de vue du retour à l'emploi puisqu'ils négligent le fait que locataires et propriétaires ne sont pas deux groupes homogènes. Au contraire, propriétaires et locataires se distinguent par des caractéristiques susceptibles d'influencer les résultats de la recherche d'emploi dont il est nécessaire de contrôler les effets par une analyse économétrique.

3. Les sorties vers l'inactivité sont donc considérées comme censurées, tout comme le faisaient Munch et al. (2006) par exemple.

4. L'application du test de Prentice (1978) et Peto \& Peto (1972) aux données françaises rejette l'hypothèse d'égalité des fonctions de survie entre propriétaires et locataires alors qu'elle l'accepte sur les données américaines. 


\subsection{Caractéristiques des individus au chômage}

Par exemple, le Tableau 2 pour la France et le Tableau 3 pour les Etats-Unis montrent que la structure par âge est très différenciée en fonction du statut résidentiel. A partir du $P E M$, 90\% des individus propriétaires ont plus de 30 ans, tandis que l'on observe l'inverse pour $63 \%$ des locataires. Il n'est donc pas surprenant que ces derniers soient plus largement caractérisés par la vie en couple sans enfants (44\%), puis par le célibat (20\%). On constate aussi que les individus sont d'autant plus représentés dans l'échantillon des chômeurs que leur niveau de diplôme est bas : presque un tiers de l'échantillon n'a aucun diplôme ou brevet, et $30 \%$ un diplôme d'enseignement technique court. A cet égard, on remarque que les propriétaires sont sur-représentés aux niveaux d'éducation les plus bas. Sur données américaines, on retrouve que les propriétaires sont plus âgés que les locataires, mais il y a peu de différences entre ces deux groupes en termes de niveau d'éducation. Il apparaît aussi un effet de l'origine ethnique aux Etats-Unis : les Blancs ont davantage de chances d'être propriétaires que les autres.

\section{Modélisation économétrique}

Pour tester l'hypothèse d'Oswald, il est insuffisant de se contenter d'introduire une variable dichotomique représentant le statut résidentiel comme variable explicative des transitions du chômage vers l'emploi. Il convient en effet de tenir compte de l'endogénéité du choix de statut dans le logement, du fait de l'existence d'interactions bidirectionnelles entre les parcours professionnels et les choix résidentiels des individus. Par exemple, le retour à l'emploi n'est parfois possible que sous condition de mobilité résidentielle, et inversement l'accession à la propriété ne peut se faire que si les individus ont une situation professionnelle suffisamment stable et non marquée par des épisodes de chômage récurrents.

Green \& Hendershott (2001) et Brunet \& Lesueur (2009, 2004) essaient de résoudre le problème d'endogénéité en utilisant une méthode d'estimation par variables instrumentales en deux étapes : elle consiste à estimer dans un premier temps une équation de statut résidentiel par un probit ou un logit et dans un second temps à utiliser la valeur estimée de la probabilité de choix du statut résidentiel dans le modèle de durée de chômage. La première amélioration que l'on propose ici est d'estimer simultanément ces deux équations par maximum de vraisemblance à information complète. La deuxième limite de ces études est qu'elles sont basées sur des estimations paramétriques des durées de chômage. Green \& Hendershott (2001) utilisent un modèle de durée fondé sur une distribution de type Weibull, qui impose à la probabilité de sortie du chômage d'être monotone avec la durée de l'épisode, hypothèse forte et peu réaliste. Même si Brunet \& Lesueur (2009, 2004) et Brunet et al. (2007) mettent en concurrence différentes estimations paramétriques et retiennent des distributions plus générales (lognormale, gamma généralisée), les spécifications non-paramétriques sont a priori plus robustes [Meyer (1990), Han \& Hausman (1990)]. C'est pourquoi, on optera pour une spécification flexible semi-paramétrique d'un modèle de hasard proportionnel. Enfin, on veut éviter d'asso- 


\begin{tabular}{|c|c|c|c|}
\hline Variables binaires & Propriétaires & Locataires & Total \\
\hline Statut résidentiel & 0,44 & 0,56 & - \\
\hline Femme & 0,62 & 0,54 & 0,57 \\
\hline Nationalité étrangère & 0,05 & 0,05 & 0,05 \\
\hline \multicolumn{4}{|l|}{ Structure familiale } \\
\hline Couple sans enfant & 0,23 & 0,44 & 0.35 \\
\hline Couple avec enfant & 0,66 & 0,31 & 0,47 \\
\hline Famille monoparentale & 0,03 & 0,05 & 0,04 \\
\hline Personne seule & 0,07 & 0,20 & 0,14 \\
\hline \multicolumn{4}{|l|}{ Statut $d u$ conjoint } \\
\hline Conjoint employé & 0,69 & 0,54 & 0,60 \\
\hline Conjoint chômeur & 0,07 & 0,12 & 0,10 \\
\hline Conjoint inactif & 0,13 & 0,08 & 0,10 \\
\hline NA ou non renseigné & 0,11 & 0,26 & 0,20 \\
\hline \multicolumn{4}{|l|}{ Âge } \\
\hline $17-29$ ans & 0,10 & 0,63 & 0,39 \\
\hline $30-39$ ans & 0,31 & 0,23 & 0,26 \\
\hline $40-49$ ans & 0,36 & 0,10 & 0,22 \\
\hline $50-60$ ans & 0,22 & 0,04 & 0,12 \\
\hline \multicolumn{4}{|l|}{ Diplôme } \\
\hline Aucun diplôme ou brevet & 0,37 & 0,27 & 0,32 \\
\hline Ens. Technique court & 0,34 & 0,27 & 0,30 \\
\hline Ens. Tech. long, Bac.gén. & 0,16 & 0,21 & 0,19 \\
\hline Ens. Sup. court & 0,08 & 0,14 & 0,11 \\
\hline Ens. Sup. long & 0,05 & 0,10 & 0,08 \\
\hline \multicolumn{4}{|l|}{ Taille d'unité urbaine } \\
\hline Commune rurale & 0,41 & 0,21 & 0,30 \\
\hline$<5000$ habitants & 0,05 & 0,05 & 0,05 \\
\hline$[5000-10000[$ habitants & 0,05 & 0,06 & 0,06 \\
\hline$[10000-20000[$ habitants & 0,05 & 0,07 & 0,06 \\
\hline$[20000-50000[$ habitants & 0,05 & 0,07 & 0,06 \\
\hline$[50000-100000[$ habitants & 0,05 & 0,08 & 0,06 \\
\hline$[100000-200000[$ habitants & 0,07 & 0,09 & 0,08 \\
\hline$[200000-2000000[$ habitants & 0,16 & 0,26 & 0,21 \\
\hline Agglomération parisienne & 0,10 & 0,11 & 0,11 \\
\hline Héritage ou donation & 0,06 & 0,04 & 0,05 \\
\hline \multicolumn{4}{|l|}{ Taxes locales } \\
\hline Taxes locales $[0-370[$ & 0,19 & 0,22 & 0,21 \\
\hline Taxes locales $[370-470[$ & 0,24 & 0,17 & 0,20 \\
\hline Taxes locales $[470-580[$ & 0,24 & 0,19 & 0,21 \\
\hline Taxes locales $[580-750[$ & 0,18 & 0,22 & 0,20 \\
\hline Taxes locales [750 et +[ & 0,15 & 0,21 & 0,18 \\
\hline Nombre d'individus & 924 & 1163 & 2028 \\
\hline
\end{tabular}

TABlEAU 2 - Caractéristiques moyennes des individus au chômage (données françaises)

cier à la propriété immobilière une influence causale sur les sorties du chômage, qui ne serait en fait que le reflet de caractéristiques individuelles inobservées. En particulier, on peut sus- 


\begin{tabular}{lccc}
\hline \hline Variables binaires & Propriétaires & Locataires & Total \\
\hline Statut résidentiel & 0,47 & 0,53 & - \\
Femme & 0,56 & 0,57 & 0,56 \\
Origine Ethnique & & & \\
$\quad$ Blanc & 0,65 & 0,47 & 0,55 \\
$\quad$ Noir & 0,30 & 0,49 & 0,40 \\
$\quad$ Autres & 0,05 & 0,04 & 0,05 \\
Statut du conjoint & & & \\
Conjoint employé & 0,61 & 0,31 & 0,45 \\
Conjoint chômeur & 0,03 & 0,03 & 0,03 \\
Conjoint inactif & 0,08 & 0,06 & 0,07 \\
NA ou non renseigné & 0,28 & 0,60 & 0,45 \\
Âge & & & \\
17-25 ans & 0,09 & 0,22 & 0,15 \\
$26-35$ ans & 0,25 & 0,40 & 0,33 \\
36-45 ans & 0,42 & 0,30 & 0,35 \\
$46-65$ ans & 0,25 & 0,09 & 0,16 \\
Diplôme & & & \\
Aucun diplôme & 0,12 & 0,19 & 0,16 \\
Diplômes de fin d'études secondaires & 0,63 & 0,64 & 0,64 \\
Diplôme d'enseignement supérieur - 1er cycle & 0,18 & 0,12 & 0,15 \\
Diplôme d'enseignement supérieur - 2ème cycle & 0,07 & 0,05 & 0,06 \\
Nombre d'individus & 649 & 720 & 1369 \\
\hline \hline
\end{tabular}

TABleau 3 - Caractéristiques moyennes des individus au chômage (données américaines)

pecter que des variables non observables telles que la préférence pour le présent, les aptitudes innées ou le degré d'efficacité dans la prospection expliquent à la fois le statut résidentiel et la durée dans l'état de chômage. Green \& Hendershott (2001) dans leur étude américaine n'en tenaient pas compte. Brunet \& Lesueur (2004, 2007, 2009) intégraient un terme de correction de cette hétérogénéité inobservée par une loi Gamma. De nouveau, on préfèrera ici recourir à une caractérisation non- paramétrique en supposant que le terme d'hétérogénéité suit une distribution discrète. Ainsi, on effectue au final une estimation semi-paramétrique d'un modèle de hasard proportionnel avec hétérogénéité inobservée et une seule sortie possible : le retour à l'emploi $(e)$. Les autres sorties possibles, c'est-à-dire vers l'inactivité, seront traitées comme des observations censurées.

\subsection{Spécification}

Plus précisément, on modélise ici le taux de hasard $\lambda_{e}$, correspondant au taux instantané de retour à l'emploi d'un chômeur sachant qu'il est resté en situation de chômage au moins jusqu'en $T$ :

$$
\lambda_{e}(t)=\lim _{d t \rightarrow 0} \frac{P(t<T \leq t+d t \mid T>t)}{d t} .
$$


Cette fonction de hasard est supposée proportionnelle, c'est-à-dire caractérisée par le produit de deux éléments : i) une fonction qui dépend des caractéristiques individuelles observables $X_{t}$, d'une dichotomique reflétant la propriété immobilière $z_{t}$ et de caractéristiques inobservées $v_{e}$ et ii) le hasard de base $\lambda_{0 e}$ qui correspond au taux de sortie du chômage commun aux individus (indépendant de leurs caractéristiques individuelles) et capte donc la dépendance temporelle du taux de hasard :

$$
\lambda_{e}(t)=\exp \left[X_{t} \beta_{e}+z_{t} \gamma_{e}+v_{e}\right] \times \lambda_{0 e}(t)
$$

On choisit de représenter $\lambda_{0 e}$ par une spécification flexible non-paramétrique de type "constante par morceaux" (piecewise constant) où les durées de chômage sont groupées en 6 intervalles : $\lambda_{0 e}(t)=\theta_{l} \times 1_{\left.\left.\{t \in] \tau_{l}, \tau_{l+1}\right\}\right\}}$ avec $l=0, \ldots, 5, \tau_{0}=0, \tau_{6}=\infty$ et $1_{\{\}}$la fonction indicatrice prenant la valeur 1 si l'expression entre parenthèses est vraie et 0 sinon.

Pour tenir compte de la potentielle endogénéité de la variable de propriété immobilière $z_{t}$, la probabilité d'être propriétaire est modélisée simultanément avec le taux précédent de sortie du chômage. On suppose que cette probabilité peut être caractérisée par le modèle logit suivant :

$$
P\left(z_{t}=1 \mid Y_{t}, v_{h}\right)=\frac{\exp \left(Y_{t} \delta_{h}+v_{h}\right)}{1+\exp \left(Y_{t} \delta_{h}+v_{h}\right)}
$$

avec $z_{t}$ qui vaut 1 si le chômeur est propriétaire de son logement en $t$ et 0 s'il en est locataire. $Y_{t}$ est l'ensemble des variables explicatives du statut résidentiel qui comprend les variables $X_{t}$ et des variables supplémentaires qui faciliteront l'identification. $v_{h}$ correspond à une composante inobservée permettant de capter l'hétérogénéité inobservée.

Les individus ayant opté pour la propriété immobilière comme mode d'occupation de leur logement sont a priori susceptibles d'être caractérisés par une plus grande tendance à la stabilité, ou par des anticipations optimistes, dues éventuellement à de meilleures capacités, quant à leurs perspectives sur le marché de l'emploi. Si les caractéristiques observables qui conditionnent le choix du statut résidentiel ne capturent pas complètement ce processus de sélection des individus dans la propriété immobilière, les variables omises représentées par le terme d'hétérogénéité seront corrélées avec les caractéristiques inobservées de l'équation de durée de chômage. On suppose que toute l'hétérogénéité individuelle inobservée est prise en compte par les termes $v_{e}, v_{h}$, et que leur corrélation restitue l'ensemble des variations simultanées inexpliquées par les caractéristiques observables entre les décisions de transition sur le marché de l'emploi et les choix de statut résidentiel. En pratique, il est donc nécessaire de caractériser la distribution jointe $G($.$) des termes \left(v_{e}, v_{h}\right)$. Pour ce faire, plusieurs approches ont été proposées dans la littérature économétrique. On retient à la suite de Belzil \& Hansen (2002) une adaptation de l'approche non-paramétrique développée par Heckman \& Singer (1984) et utilisée dans les travaux récents étudiant l'impact du statut résidentiel sur la durée de chômage dans d'autres pays [Munch et al. (2006), Battu et al. (2008), van Vuuren (2009)]. On choisit ainsi une distribution non paramétrique des termes d'hétérogénéité en supposant que les individus sont répartis selon une probabilité $p$ en deux types particuliers définis par un vecteur de points 
de support : $v_{1}=\left(v_{e}^{1}, v_{h}^{1}\right)$ avec la probabilité $p=\operatorname{Pr}\left(v_{e}=v_{e}^{1}, v_{h}=v_{h}^{1}\right)$ et $v_{2}=\left(v_{e}^{2}, v_{h}^{2}\right)$ avec la probabilité $(1-p)$. Enfin, l'un des points de support doit être normalisé à zéro $\left(v_{2}=(0,0)\right)$ car le taux de hasard de base agit comme un terme constant sur les taux de $\operatorname{sorties}^{5}$.

\subsection{Estimation}

L'estimation conjointe des équations (2) et (3) peut être réalisée par maximum de vraisemblance, une fois celle-ci construite. La fonction de vraisemblance globale s'écrit à partir des vraisemblances associées à chacune des équations du modèle. Premièrement, la log-vraisemblance associée au modèle de durée de chômage tient compte non seulement des sorties vers l'emploi mais aussi des épisodes de chômage censurés - via la probabilité de survie dans l'état de chômage jusqu'en $T$ (2ème terme) :

$$
l l_{t}\left(\beta_{e}, \theta_{l}, \gamma_{e} \mid t, X_{t}, z_{t}, v_{e}\right)=\ln \lambda_{e}(t)-\int_{0}^{t} \theta_{e}(s) d s
$$

Deuxièmement, la log-vraisemblance correspondante aux choix de statut résidentiel découle directement du logit présenté à l'équation (3) :

$$
l l_{h}\left(\beta_{h}, \delta_{h} \mid z_{t}, Y_{t}, v_{h}\right)=\sum_{t} z_{t} \ln \left(P\left(z_{t}=1 \mid Y_{t}, v_{h}\right)\right)+\left(1-z_{t}\right) \ln \left(1-P\left(z_{t}=1 \mid Y_{t}, v_{h}\right)\right)
$$

La vraisemblance jointe s'écrit comme le produit des vraisemblances associées au modèle de durée de chômage et au choix de statut résidentiel (équations 4 et 5), étant donnée la distribution discrète de l'hétérogénéité inobservée, le tout intégré sur l'ensemble de la distribution $G\left(v_{e}, v_{h}\right)$. Du fait de la présence dans l'échantillon d'épisodes multiples de chômage, la log-vraisemblance jointe pour un individu $i$ a pour expression :

$$
l l_{t, h, i}=\ln \int_{v_{e}} \int_{v_{h}} \prod_{j=1}^{N_{i}} \exp \left[l l_{t_{j}}\left(\beta_{e}, \theta_{l}, \gamma_{e} \mid t, X_{t}, z_{t}, v_{e}\right)+l l_{h_{j}}\left(\beta_{h}, \delta_{h} \mid z_{t}, Y_{t}, v_{h}\right)\right] d G\left(v_{e}, v_{h}\right),
$$

où $j$ représente l'épisode $j$ et $N_{i}$ le nombre total d'épisodes de chômage pour l'individu $i$. Au final, la log-vraisemblance à maximiser pour estimer les paramètres associés aux variables explicatives et les paramètres d'hétérogénéité individuelle est égale à la somme des $\log$-vraisemblances individuelles, soit $\ln L=\sum_{i} l l_{t, h, i}$.

\subsection{Identification}

Les variables explicatives retenues dans les équations de durées de chômage $\left(X_{t}\right)$ et dans l'équation du choix du statut résidentiel $\left(Y_{t}\right)$ sont relativement similaires à celles utilisées dans

5. Pour plus de détails sur cette modélisation, voir par exemple van den Berg (2001). 
les études de Munch et al. (2006), Barceló (2006), Battu et al. (2008) et van Vuuren (2009). $X$ incorpore des caractéristiques démographiques relatives à l'individu (sexe, âge, nationalité/race, diplôme) et à son ménage (structure familiale, statut du conjoint, taille de l'unité urbaine de résidence). $Y$ intègre l'ensemble des variables $X$, plus des variables de quantiles de taxes locales (taxe foncière et taxe d'habitation) du lieu de résidence, calculées par taille d'unité urbaine et département pour le $P E M$ et par état (faute d'informations géographiques plus fines) pour le PSID. On espère à travers ces variables représenter les aménités liées au lieu de résidence, qui sont censées augmenter la probabilité d'être propriétaire de son logement ${ }^{6}$ sans pour autant modifier les probabilités de transition sur le marché du travail. Par ailleurs, ces variables additionnelles vont servir de conditions d'exclusion améliorant l'identification de l'influence du statut résidentiel sur la durée de chômage.

Comme dans Munch et al. (2006), Barceló (2006), Battu et al. (2008) et van Vuuren (2009), l'identification de l'effet de la propriété immobilière est assurée ici par les épisodes multiples de chômage pour un nombre suffisant d'individus. Les données utilisées présentent l'avantage de suivre les individus dans le temps, tant au niveau de leurs transitions sur le marché du travail qu'à celui des changements de mode résidentiel. Ainsi, on a des épisodes multiples de chômage pour un même individu et pour certains d'entre eux leur statut résidentiel varie entre ces épisodes. Il est aussi possible d'observer pour un même individu des changements de mode d'occupation du logement au cours d'un épisode de chômage particulier. Sous ces conditions, van den Berg (2001) et Abbring \& van den Berg (2003) montrent que l'effet du "traitement", c'est-à-dire ici de la propriété immobilière, est identifié sans avoir à trouver d'instruments. L'ajout des variables de taxes locales dans l'équation de statut résidentiel ne peut que renforcer cette identification mais n'était pas indispensable.

\section{Les résultats empiriques}

Les tableaux 4 et 5 présentent les résultats, respectivement sur données françaises et sur données américaines, de l'estimation conjointe de l'équation du taux de sortie du chômage et du choix de statut résidentiel. On commencera par analyser succinctement les déterminants individuels du statut résidentiel et des taux de sorties vers l'emploi afin de vérifier s'ils sont cohérents avec ceux trouvés traditionnellement dans la littérature. Puis, on examinera notre question principale à savoir les liens entre statut résidentiel et durée de chômage.

\subsection{Les déterminants du statut résidentiel}

L'impact des différentes variables explicatives sur le choix de la propriété immobilière comme statut d'occupation est sensiblement le même sur données américaines et sur données françaises. On commentera donc les résultats obtenus de façon globale. On trouve la plupart

6. Cette variable capte également un effet de coût du logement. 


\begin{tabular}{|c|c|c|c|c|}
\hline & \multicolumn{2}{|c|}{$\begin{array}{l}\text { Taux de sortie du chômage } \\
\lambda_{e}(t)\end{array}$} & \multicolumn{2}{|c|}{$\begin{array}{c}\text { Proba. d'être propriétaire } \\
\qquad P\left(z_{t}=1\right)\end{array}$} \\
\hline & Coef.estimé & Ec.-type & Coef.estimé & Ec.-type \\
\hline Propriétaire & $0,176^{*}$ & $(0,10)$ & & \\
\hline Femme & $-0,311^{* * *}$ & $(0,04)$ & $0,501 * * *$ & $(0,10)$ \\
\hline Nationalité étrangère & $-0,050$ & $(0,10)$ & $-0,187$ & $(0,15)$ \\
\hline \multicolumn{5}{|c|}{ Struct. fam. (Réf. : couple sans enfant) } \\
\hline Couple avec enfant & $-0,169 * * *$ & $(0,05)$ & $0,398^{* * *}$ & $(0,09)$ \\
\hline Famille mono-parentale & $-0,142$ & $(0,11)$ & $-0,924^{* * *}$ & $(0,24)$ \\
\hline Personne seule & $-0,005$ & $(0,07)$ & $-0,914^{* * *}$ & $(0,22)$ \\
\hline \multicolumn{5}{|c|}{ Statut conjoint (Réf. : conjoint employé) } \\
\hline Conjoint chômeur & 0,059 & $(0,07)$ & $-0,562 * * *$ & $(0,14)$ \\
\hline Conjoint inactif & $-0,025$ & $(0,07)$ & $-0,096$ & $(0,10)$ \\
\hline \multicolumn{5}{|l|}{ Âge $(17-29$ ans $)$} \\
\hline $30-39$ ans & $-0,337^{* * *}$ & $(0,06)$ & $1,768^{* * *}$ & $(0,39)$ \\
\hline 40-49 ans & $-0,491 * * *$ & $(0,08)$ & $2,497^{* * *}$ & $(0,48)$ \\
\hline $50-60$ ans & $-1,306 * * *$ & $(0,11)$ & $3,005^{* * *}$ & $(0,53)$ \\
\hline \multicolumn{5}{|c|}{ Diplôme (Réf. : aucun ou primaire) } \\
\hline Ens. Technique court & $0,119 * *$ & $(0,05)$ & $0,210^{* * *}$ & $(0,08)$ \\
\hline Ens. Tech. long,Bac.gén. & $0,154^{* *}$ & $(0,06)$ & $0,193^{* *}$ & $(0,09)$ \\
\hline Ens. Sup. court & $0,265 * * *$ & $(0,07)$ & 0,043 & $(0,12)$ \\
\hline Ens. Sup. long & 0,071 & $(0,08)$ & $-0,151$ & $(0,14)$ \\
\hline \multicolumn{5}{|c|}{ Taille d'unité urbaine (Réf. : rurale) } \\
\hline$<5000$ habitants & $-0,110$ & $(0,09)$ & $-0,257^{*}$ & $(0,14)$ \\
\hline [5000 - 10000[ habitants & $-0,041$ & $(0,09)$ & $-0,578^{* * *}$ & $(0,16)$ \\
\hline$[10000-20000[$ habitants & $-0,089$ & $(0,09)$ & $-0,721^{* * *}$ & $(0,16)$ \\
\hline$[20000-50000[$ habitants & $-0,118$ & $(0,09)$ & $-0,394^{* * *}$ & $(0,15)$ \\
\hline$[50000-100000[$ habitants & $-0,148$ & $(0,09)$ & $-0,529 * * *$ & $(0,16)$ \\
\hline [100000 - 200000[ habitants & $-0,063$ & $(0,08)$ & $-0,649^{* * *}$ & $(0,14)$ \\
\hline$[100000-2000000[$ habitants & $-0,252^{* * *}$ & $(0,06)$ & $-0,682^{* * *}$ & $(0,13)$ \\
\hline Agglomération parisienne & $-0,161^{* *}$ & $(0,07)$ & $-0,431^{* * *}$ & $(0,12)$ \\
\hline Héritage ou donation & & & 0,208 & $(0,14)$ \\
\hline \multicolumn{5}{|l|}{ Taxes locales (Réf. : $[0-370[$ ) } \\
\hline Taxes locales $[370-470[$ & & & $0,183^{*}$ & $(0,10)$ \\
\hline Taxes locales $[470-580[$ & & & $0,367 * * *$ & $(0,11)$ \\
\hline Taxes locales [580 - 750[ & & & 0,163 & $(0,11)$ \\
\hline Taxes locales [750et + [ & & & 0,07 & $(0,11)$ \\
\hline Constante & & & $-2,547^{* * *}$ & $(0,63)$ \\
\hline \multicolumn{5}{|c|}{ Décomposition du hasard de base $\theta_{l}$} \\
\hline Chômage $<1$ mois & $-2,288^{* * *}$ & $(0,09)$ & $-2,236 * * *$ & $(0,10)$ \\
\hline Chômage 1-2 mois & $-1,667 * * *$ & $(0,08)$ & $-1,604^{* * *}$ & $(0,09)$ \\
\hline Chômage 3-5 mois & $-1,627^{* * *}$ & $(0,07)$ & $-1,552^{* * *}$ & $(0,08)$ \\
\hline Chômage 6-12 mois & $-2,003^{* * *}$ & $(0,08)$ & $-1,920 * * *$ & $(0,09)$ \\
\hline Chômage 1-2 ans & $-2,352^{* * *}$ & $(0,09)$ & $-2,251^{* * *}$ & $(0,10)$ \\
\hline Chômage $>2$ ans & $-2,732^{* * *}$ & $(0,11)$ & $-2,601 * * *$ & $(0,13)$ \\
\hline Hétérogénéité & $-0,475$ & $(0,41)$ & 1,870 & $(1,14)$ \\
\hline Probabilité & $p=0,893^{* * *}$ & $(0.15)$ & & \\
\hline$\chi^{2}$ & 4861,986 & & & \\
\hline Nb. d'épisodes & 3664 & & & \\
\hline Nb. d'individus & 2028 & & & \\
\hline Log-vrais. & $-10857,337$ & & & \\
\hline
\end{tabular}

TABleau 4 - Probabilité de transition vers un emploi (données françaises) 


\begin{tabular}{|c|c|c|c|c|}
\hline & \multicolumn{2}{|c|}{$\begin{array}{c}\text { Taux de sortie du chômage } \\
\lambda_{e}(t)\end{array}$} & \multicolumn{2}{|c|}{$\begin{array}{c}\text { Proba. d'être propriétaire } \\
\qquad P\left(z_{t}=1\right)\end{array}$} \\
\hline & Coef.estimé & Ec.-type & Coef.estimé & Ec.-type \\
\hline Propriétaire & $-0,205$ & $(0,16)$ & & \\
\hline Femme & $-0,106$ & $(0,08)$ & 0,090 & $(0,09)$ \\
\hline \multicolumn{5}{|c|}{ Origine ethnique (Réf. : Blanche) } \\
\hline Noire & $-0,293^{* * *}$ & $(0,09)$ & $-0,511^{* * *}$ & $(0,11)$ \\
\hline Autre & $-0,269$ & $(0,17)$ & 0,047 & $(0,21)$ \\
\hline \multicolumn{5}{|c|}{ Struct. fam. (Réf. : couple sans enfant) } \\
\hline Couple avec enfant & $-0,104$ & $(0,09)$ & $0,251^{*}$ & $(0,11)$ \\
\hline Famille mono-parentale & $-0,211$ & $(0,14)$ & $-0,739 * * *$ & $(0,19)$ \\
\hline Personne seule & $-0,162$ & $(0,12)$ & $-0,751^{* * *}$ & $(0,15)$ \\
\hline \multicolumn{5}{|c|}{ Statut conjoint (Réf. : conjoint employé) } \\
\hline Conjoint chômeur & $-0,282$ & $(0,20)$ & $-0,578^{*}$ & $(0,26)$ \\
\hline Conjoint inactif & 0,114 & $(0,14)$ & $-0,111$ & $(0,16)$ \\
\hline \multicolumn{5}{|l|}{ Âge $(17-25$ ans $)$} \\
\hline $26-35$ ans & $-0,130$ & $(0,11)$ & $0,548 * *$ & $(0,17)$ \\
\hline $36-45$ ans & $-0,098$ & $(0,11)$ & $1,216^{* * *}$ & $(0,18)$ \\
\hline $46-65$ ans & $-0,279^{*}$ & $(0,14)$ & $1,658^{* * *}$ & $(0,22)$ \\
\hline \multicolumn{5}{|l|}{ Diplôme (Réf. : Aucun) } \\
\hline Fin d'études secondaires & $0,366^{* *}$ & $(0,11)$ & 0,253 & $(0,13)$ \\
\hline Ens. Sup. 1er cycle & $0,711^{* * *}$ & $(0,14)$ & $0,420^{*}$ & $(0,16)$ \\
\hline Ens. Sup. 2ème cycle & 0,259 & $(0,17)$ & $0,475^{*}$ & $(0,21)$ \\
\hline \multicolumn{5}{|l|}{ Région (Réf. : Sud) } \\
\hline Nord Est & $-0,007$ & $(0,11)$ & 0,157 & $(0,16)$ \\
\hline Centre-Nord & $-0,073$ & $(0,09)$ & 0,049 & $(0,12)$ \\
\hline Ouest & $-0,042$ & $(0,10)$ & $-0,174$ & $(0,15)$ \\
\hline Croyance religieuse & & & 0,063 & $(0,14)$ \\
\hline \multicolumn{5}{|c|}{ Taxes locales (Réf. : 1er quantile) } \\
\hline 2ème quantile & & & 0,021 & $(0,13)$ \\
\hline 3ème quantile & & & $-0,102$ & $(0,15)$ \\
\hline 4ème quantile & & & $-0,366^{*}$ & $(0,16)$ \\
\hline 5ème quantile & & & $-0,331$ & $(0,17)$ \\
\hline Constante & & & $-0,590$ & $(0,46)$ \\
\hline \multicolumn{5}{|c|}{ Décomposition du hasard de base $\theta_{l}$} \\
\hline Chômage $<1$ mois & $-1,056 * * *$ & $(0,31)$ & & \\
\hline Chômage 1-2 mois & $-2,007 * * *$ & $(0,33)$ & & \\
\hline Chômage 3-5 mois & $-0,847^{*}$ & $(0,34)$ & & \\
\hline Chômage $6-12$ mois & $-0,758$ & $(0,39)$ & & \\
\hline Chômage 1-2 ans & $-1,257^{* *}$ & $(0,43)$ & & \\
\hline Chômage $>2$ ans & $-1,236^{* *}$ & $(0,47)$ & & \\
\hline Hétérogénéité & 1,00 & $(0,78)$ & $-1,03^{* * *}$ & $(0,52)$ \\
\hline Probabilité & $p=0,73^{* * *}$ & $(0.15)$ & & \\
\hline$\chi^{2}$ & 209,848 & & & \\
\hline Nb.d'épisodes & 1571 & & & \\
\hline Nb.d'individus & 1369 & & & \\
\hline Log-vrais. & $-4035,778$ & & & \\
\hline
\end{tabular}

TABleau 5 - Probabilité de transition vers un emploi (données américaines)

des effets attendus des variables explicatives. Par rapport aux individus en couple sans enfants, les couples avec enfants sont de manière significative plus susceptibles d'avoir opté pour la propriété. On observe l'inverse pour les familles monoparentales et les célibataires, conformément 
à la littérature empirique. Notamment du fait d'un effet richesse, les ménages composés de deux membres en emploi ont plus de chances d'être propriétaires de leur résidence principale que les couples avec un conjoint au chômage, toutes choses étant égales par ailleurs. Les effets des variables d'âge sont également en conformité avec les prédictions des théories de cycle de vie : ceteris paribus la probabilité d'être propriétaire augmente avec l'âge.

A partir du PEM, les effets des variables d'éducation indiquent que les individus titulaires d'un diplôme de l'enseignement technique (court ou long) ou du baccalauréat général ont relativement plus de chances d'être propriétaires de leur logement que ceux qui n'ont aucun diplôme. En revanche, on n'observe pas d'impact significatif pour les catégories de diplôme les plus élevées en France contrairement aux Etats-Unis. Une autre différence entre les deux pays est que les femmes françaises auraient, toutes choses étant égales par ailleurs une probabilité plus élevée d'avoir choisi la propriété [une régularité empirique obtenue également par Munch et al. (2006) et van Vuuren (2009)] contrairement aux femmes américaines. Aux Etats-Unis, ce serait davantage l'origine ethnique de l'individu plutôt que son sexe qui serait déterminant : les Noirs ont moins de chances, ceteris paribus, d'accéder à la propriété. En France, l'impact du type d'unité urbaine dans lequel l'individu réside est significativement négatif et cet effet augmente tendanciellement en valeur absolue avec le nombre d'habitants de l'unité urbaine.

Enfin, sur l'échantillon du PEM, les variables représentant les montants des taxes locales, dont on interprète les effets comme ceux des aménités liées au lieu de résidence, ont bien un effet significatif positif pour ce qui concerne la deuxième et troisième classe au-delà de la référence. Sur données américaines, seule la troisième classe de taxes locales est statistiquement significative. L'identification de l'effet du statut résidentiel dans notre modèle conjoint se fait donc principalement par les épisodes multiples.

$\mathrm{Au}$ final, les résultats obtenus sur les déterminants du statut résidentiel en France et aux Etats-Unis sont conformes à la littérature empirique sur le sujet.

\subsection{Les déterminants des taux de sortie vers l'emploi}

Regardons maintenant s'il en est de même pour les déterminants des taux de sortie vers l'emploi et s'il existe une différence entre la France et les Etats-Unis.

De manière générale, on trouve que les variables influençant les durées de chômage en France sont très similaires à celles jouant aux Etats-Unis, et ce malgré les fortes divergences dans les caractéristiques et le fonctionnement des marchés du travail de ces deux pays. Selon les travaux empiriques sur les durées de chômage en France [par exemple, Bonnal \& Fougère (1990), Bonnal, Fougère \& Sérandon (1994), Cases \& Lollivier (1994), Bonnal, Fougère \& Sérandon (1997), Magnac (2000)], on s'attend à ce que les femmes, les individus de nationalité étrangère et les jeunes aient plus fréquemment des difficultés de ré-insertion sur le marché du travail et expérimentent des durées de chômage plus longues. En revanche, la détention 
d'un diplôme devrait favoriser les sorties du chômage, sans pour autant qu'il existe une stricte hiérarchie entre les différents niveaux d'éducation.

Les résultats des tableaux 4 et 5 confortent globalement ces prédictions. Premièrement, en France, l'âge de l'individu diminue progressivement et de façon de plus en plus marquée les retours en emploi. Aux Etats-Unis, les chômeurs de plus de 45 ans ont davantage de difficultés à retrouver un emploi que leurs homologues plus jeunes. Ces observations sont aussi en accord avec celles recensées dans les études récentes [Barceló (2006), Munch et al. (2006) ou van Vuuren (2009)]. Deuxièmement, les femmes françaises ont des taux de transition vers l'emploi significativement plus faibles que les hommes, résultat de nouveau similaire à Barceló (2006), Munch et al. (2006) ou van Vuuren (2009). Aux Etats-Unis, le fait d'être une femme n'accroît pas les durées de chômage contrairement au fait d'être de race noire. La structure familiale semble peu influencer les probabilités de transition vers un emploi. Seul le fait d'avoir des enfants au sein du couple diminue significativement le retour à l'emploi en France.

A partir des données du PSID et du PEM, les chômeurs possédant un diplôme ont plus de chances de trouver un emploi que les non-diplômés, hormis ceux avec les plus hauts niveaux de diplôme, peut-être à cause de compétences trop pointues et plus difficilement modulables rapidement. Ce résultat est donc relativement conforme à la littérature sur les dotations en capital humain des individus [et par exemple aux effets des variables d'éducation dans Munch et al. (2006)].

Enfin, on peut noter que les coefficients associés à la décomposition du hasard de base sont négatifs, signifiant que la durée passée au chômage réduit la probabilité d'en sortir. On trouve ici une caractérisation des effets de stigmate associés au chômage de longue durée, de la dévalorisation du capital humain, ou du découragement des chercheurs d'emploi. En conséquence, nos estimations des probabilités de fin des épisodes de chômage indiquent peu de différences entre France et Etats-Unis et ont des résultats cohérents avec la littérature empirique.

\subsection{L'impact du statut résidentiel sur les durées de chômage}

Au-delà des déterminants traditionnels du statut résidentiel et des taux de sortie du chômage, il nous reste à répondre à notre question principale sur les liens entre propriété immobilière et durée de chômage. L'examen des coefficients associés à la variable de propriété immobilière dans les tableaux 4 et 5 permet de savoir si les locataires et les propriétaires ont un rapport différencié au chômage, en France et aux Etats-Unis.

Sur l'échantillon américain, la variable de propriété immobilière n'a pas d'impact statistiquement significatif sur les taux de transition des chômeurs vers l'emploi. Ainsi même après avoir tenu compte de l'hétérogénéité individuelle observée et inobservée entre propriétaires et locataires, on retrouve des conclusions similaires à notre analyse descriptive de type KaplanMeier (Figure 3). Ce résultat contredit la précédente étude menée par Green \& Hendershott 
(2001) pour les Etats-Unis. Ces derniers mettaient en évidence un effet négatif de la propriété immobilière sur les sorties du chômage puisqu'elle était synonyme d'allongement des durées de chômage. Toutefois, leur modélisation économétrique était contestable sur de nombreux points : i) leur modèle de durée était fondé sur la distribution Weibull; ii) l'identification de l'effet du statut résidentiel était réalisée par l'utilisation de variables dichotomiques caractérisant chaque état américain comme instruments, ce qui est fortement discutable; iii) l'hétérogénéité des caractéristiques inobservées n'était pas prise en compte. En conséquence, on pense que l'on doit accorder un crédit limité aux résultats de Green \& Hendershott (2001) et qu'en revanche nos résultats pourraient désormais servir de référence pour le cas américain.

Plusieurs justifications peuvent être avancées pour expliquer cette non-significativité de la propriété immobilière sur la durée de chômage. D'une part, le taux de chômage aux Etats-Unis est beaucoup plus faible et le marché du travail se caractérise par une plus grande flexibilité que dans la plupart des pays européens. D'autre part, les transactions pour changer de logement sont relativement plus aisées aux Etats-Unis puisque les droits de mutation sont vraiment plus faibles. Cela pourrait alors limiter les effets néfastes de la propriété immobilière sur les retours en emploi suggérés par Oswald, y compris quand ils nécessitent une mobilité résidentielle. Ainsi, il n'est pas illogique que les parcours sur le marché du travail (du moins dans la dimension durée de chômage qui nous intéresse ici) et du logement soient relativement peu interdépendants. Les statuts résidentiels ne semblent pas au final jouer un rôle majeur dans les mécanismes d'ajustement de l'emploi sur le marché du travail américain. A ce titre l'impact des contraintes de coûts de déplacement quotidiens entre centre ville et périphérie qui nourrit la thèse du spatial mismatch, semblerait plus prégnante pour apporter une explication robuste à la dispersion des durées de chômage pour les Etats-Unis [Zenou (2009)].

Parmi les travaux empiriques, soucieux de tenir compte adéquatement de l'endogénéité et de l'hétérogénéité inobservée, cette absence de différence marquée dans les durées de chômage entre propriétaires et locataires avait déjà été mise en évidence par Battu et al. (2008) pour le Royaume-Uni, pays dans lequel les conditions de fonctionnement du marché du travail relèvent comme aux Etats-Unis d'une logique plus concurrentielle avec une forte dispersion des salaires et un niveau de taux de chômage relativement faible.

Une hypothèse additionnelle permettant de rendre compte de l'absence d'effet du statut de propriétaire sur la durée de chômage aux Etats-Unis et au Royaume-Uni peut aussi être avancée si l'on note les similitudes entre ces deux pays concernant les conditions de financement de l'accession à la propriété. Dans un contexte de secteurs bancaires relativement moins régulés qu'en France, les conditions d'accès aux prêts immobiliers sont moins restrictives et la titrisation des prêts permet une flexibilité accrue dans les transactions sur le marché du logement. Les effets de contagion sur le marché du travail à travers les restrictions à la mobilité que la propriété immobilière induit sont ainsi relativement neutralisés (nonobstant la question éminemment problématique de la soutenabilité d'un tel fonctionnement).

La situation apparaît toute autre en France. La variable de statut résidentiel est statistiquement significative (à un seuil de risque de 8\%) suggérant que la propriété immobilière a bien 
un impact direct sur les transitions chômage-emploi. Son coefficient dans la fonction de hasard étant positif, les propriétaires français au chômage retrouveraient ainsi un emploi, toutes choses étant égales par ailleurs, plus rapidement que leurs homologues locataires. Ce résultat va dans le sens inverse de ce que pouvait laisser entendre l'analyse descriptive de la Figure 3. Cependant, ils ne sont pas contradictoires : ils relèvent simplement que les propriétaires, notamment plus âgés, moins diplômés et vivant plus majoritairement avec un conjoint en emploi, ont des durées effectives de chômage plus longues. Mais, entre un locataire et un propriétaire qui présenteraient exactement toutes les mêmes caractéristiques, le chômeur propriétaire aurait des chances beaucoup plus importantes de sortir rapidement du chômage que son homologue locataire. Selon nos estimations, la propriété immobilière augmenterait la probabilité de retour à l'emploi d'environ $19 \%$.

A titre de comparaison, Munch et al. (2006) pour le Danemark et van Vuuren (2009) pour les Pays-Bas avaient déjà trouvé, avec des méthodologies économétriques identiques, que les propriétaires avaient des durées de chômage plus faibles que les locataires ceteris paribus. Néanmoins, l'avantage conféré par la propriété immobilière pour sortir du chômage semble plus marqué au Danemark qu'en France : Munch et al. (2006) ont évalué que les propriétaires ont une probabilité de retrouver un emploi supérieure de $41 \%$ à celle des locataires. Par rapport aux travaux déjà existants pour la France, notre estimation confirme partiellement les résultats de Brunet et al. (2007). Avec une décomposition plus fine des statuts résidentiels qu'il n'a pas été possible de retenir dans notre étude comparative, ces derniers montraient que, pour la France, la propriété immobilière augmentait la durée de chômage pour les propriétaires de plein droit, mais diminuait également de manière très significative celle des propriétaires accédants. On peut en effet supposer que les accédants à la propriété dont les charges d'emprunt rendent les coûts de mobilité plus élevés, auraient tendance à manifester lors de leur prospection des salaires de réserves plus bas, d'une part pour accélérer leur sortie du chômage en période d'endettement, et d'autre part pour retrouver un emploi à proximité d'un domicile récemment acquis dont ils souhaitent conserver les aménités. Ne pas supporter les coûts de délocalisation des autres membres du ménage est un autre élément qui milite en faveur d'une réduction du rayon de recherche d'emploi et d'un salaire de réserve plus bas de la part des accédants. Or, en France, environ deux tiers des propriétaires ont encore des prêts immobiliers à rembourser et sont donc considérés comme accédants. Il n'est pas donc surprenant que dans notre étude où la catégorie des propriétaires regroupe à la fois les propriétaires de pleins droits et les accédants à la propriété, par un simple effet de structure, l'effet des accédants ait pu en moyenne l'emporter.

Si tel est le cas, la propriété immobilière ne serait pas à complètement associer à une meilleure qualité de l'insertion professionnelle en France. Un retour rapide à l'emploi pourrait être synonyme de mauvais appariement. Les propriétaires ne réduiraient alors la durée des épisodes de chômage qu'au détriment de leur salaire, ce qui serait néfaste en termes de productivités individuelles et d'efficacité de la gestion de la main d'œuvre. Sur ce sujet, Brunet \& Havet (2009) ont mis en évidence sur les données du PEM pour la France, que parmi la population en emploi, les propriétaires sont davantage déclassés à la fois en termes de salaires et de postes occupés que les locataires. En liaison avec nos résultats, une analyse plus fine de 
la déqualification, ciblée sur les salariés qui sortent d'un épisode de chômage, pourrait être fort instructive pour renforcer les intuitions relatives au problème de défaut d'appariement. $\mathrm{Au}$ delà de ce prolongement, le contrôle des facteurs subjectifs de satisfaction dans le logement permettrait peut-être de poursuivre l'analyse de l'imbrication étroite entre choix résidentiels et décisions en matière d'emploi. Le déclassement salarial dont semble souffrir les propriétaires plus fortement que les locataires serait alors représentatif d'une logique inspirée d'une théorie hédonique des salaires « étendue » selon laquelle pour les propriétaires, les aménités associées au logement compenseraient la perte de satisfaction liée au déclassement salarial.

\section{Conclusion}

Cet article s'inscrit dans la lignée des travaux microéconométriques qui cherchent à tester empiriquement la pertinence de « l'hypothèse d'Oswald $\gg$, selon laquelle la propriété immobilière est un obstacle pour sortir du chômage. En mobilisant les données françaises du Panel Européen des Ménages et les données américaines du Panel Study of Income Dynamics pour la période 1994-2001, l'analyse des effets d'interaction entre le marché du logement et la qualité de l'insertion professionnelle est enrichie par la comparaison de deux marchés du travail très spécifiques.

On pouvait a priori s'attendre à une position des propriétaires relativement plus favorable sur le marché du travail américain que sur le marché français. En particulier, les plus forts coûts associés aux transactions immobilières en France sont susceptibles de constituer un obstacle majeur à la mobilité des propriétaires et donc à leur processus de recherche d'emploi dans un contexte de taux de chômage relativement élevé. L'analyse descriptive a en effet mis en évidence que les propriétaires restaient plus longtemps au chômage que les locataires en France mais pas aux Etats-Unis. Toutefois, la prise en compte de l'hétérogénéité observée et inobservée entre propriétaires et locataires modifie sensiblement ces conclusions.

L'estimation conjointe du statut résidentiel et d'un modèle de hasard proportionnel avec hétérogénéité inobservée non paramétrique montre que la propriété immobilière joue peu dans les mécanismes d'ajustement de l'emploi et notamment non significativement sur les durées de chômage aux Etats-Unis. En revanche, les propriétaires français au chômage retrouvent un emploi plus rapidement que leurs homologues locataires. Ce dernier résultat est donc contradictoire avec les arguments de l'hypothèse d'Oswald. Toutefois, avant de conclure que la propriété immobilière serait un atout quant à l'insertion sur le marché du travail en France, il conviendrait d'examiner la qualité des appariements avec les emplois retrouvés. Ce retour à l'emploi plus rapide des propriétaires pourrait en effet refléter le fait que les propriétaires sont davantage prêts à accepter des emplois pour lesquels leur capital humain est sous-valorisé, afin d'éviter que le retour à l'emploi nécessite une relocalisation résidentielle.

Enfin, la période couverte par nos données est particulière, puisqu'elle est marquée par une progression importante de l'accession à la propriété (en France et plus encore aux Etats- 
Unis). L'éclatement de la bulle immobilière et les modifications drastiques de l'accès au crédit pourraient bouleverser la donne dans les années à venir, particulièrement aux Etats-Unis.

\section{Références}

Abbring, J. \& van den Berg, G. (2003), 'The Nonparametric Identification of Treatment Effects in Duration Models', Econometrica 71(5), 1491-1517.

Ansieau, D., Breuil-Genier, P. \& Hourriez, J.-M. (2001), 'Le Panel européen : une source statistique longitudinale sur les revenus et les conditions de vie des ménages', Economie et Statistique 349-350(9/10), 3-15.

Barceló, C. (2006), 'Housing Tenure and Labour Mobility : A Comparison Across European Countries', DOCUMENTO DE TRABAJO, Banco De Espana 0603.

Battu, H., Ma, A. \& Phimister, E. (2008), 'Housing Tenure, Job Mobility and Unemployment in the UK', The Economic Journal 118(257), 311-328.

Belzil, C. \& Hansen, J. (2002), 'Unobserved Ability and the Return to Schooling', Econometrica 70(5), 2075-2091.

Bonnal, L. \& Fougère, D. (1990), 'Les déterminants individuels des durées du chômage', Économie et Prévision 96(5), 45-82.

Bonnal, L., Fougère, D. \& Sérandon, A. (1994), 'L'impact des dispositifs d'emploi sur le devenir des jeunes chômeurs : une évaluation économétrique sur données longitudinales', Économie et Prévision 115(4), 1-28.

Bonnal, L., Fougère, D. \& Sérandon, A. (1997), 'Evaluating the Impact of French Policies on Individual Labour Markets Histories', Review of Economic Studies 64(4), 683-713.

Bosvieux, J. (2005), 'Accession à la propriété : des acquéreurs plus nombreux moins prudents', Economie et Statistique 381-382, 41-62.

Breuil-Genier, P. \& Valdelièvre, H. (2001), 'Le Panel européen : l'intérêt d'un panel d'individus', Economie et Statistique 349-350(9/10), 17-40.

Brunet, C. \& Havet, N. (2009), 'Propriété immobilière et déqualification dans l'emploi', Revue française d'Economie 24(1), 121-155.

Brunet, C. \& Lesueur, J.-Y. (2004), 'Le statut résidentiel affecte-t-il la durée de chômage? : Applications microéconométriques sur données françaises', Revue Économique 55(3), 569-578.

Brunet, C. \& Lesueur, J.-Y. (2009), Do Homeowners Stay Unemployed Longer? Evidence Based on French Data, in C. van Ewijk \& M. van Leuvensteijn, eds, 'Homeownership \& the Labour Market in Europe', Oxford University Press, pp. 137-158.

Brunet, C., Clark, A. \& Lesueur, J.-Y. (2007), 'Statut résidentiel et durée de chômage en France et au Royaume-Uni', Revue française d'Economie 22(2), 165-190.

Buchel, F. \& van Ham, M. (2003), 'Overeducation, regional labor markets, and spatial flexibility', Journal of Urban Economics 53(3), 482-493.

Cases, C. \& Lollivier, S. (1994), 'Estimation d'un modèle de sortie de chômage à destinations multiples', Economie et Prévision 2-3(113-114), 177-188. 
Daubresse, M. (2003), 'La reprise de l'accession à la propriété', Insee Première 3(913), 4 pages.

Dubujet, F. \& Blanc, D. L. (2000), 'Accession à la propriété immobilière : le régime de croisière?', Insee Première 3(718), 4 pages.

Green, R. \& Hendershott, P. (2001), 'Home-ownership and the duration of unemployment : a test of the Oswald hypothesis', mimeo .

Han, A. \& Hausman, J. (1990), 'Flexible Parametric Estimation of Duration and Competing Risk Models', Journal of Applied Econometrics 5(1), 1-28.

Heckman, J. \& Singer, B. (1984), 'Econometric Duration Analysis', Journal of Econometrics $\mathbf{2 4}(1 / 2), 63-132$.

Laferrère, A. (2005), 'Quitter le nid : entre forces centripètes et centrifuges', Economie et Statistique 381-382, 147-175.

Magnac, T. (2000), 'Subsidised Training and Youth Employment : Distinguishing Unobserved Heterogeneity from State Dependence in Labor Market Histories', The Economic Journal 110(466), 805-837.

Manning, A. (2003), Monopsony in Motion, Princeton, New Jersey : Princeton University Press.

McGonagle, K. \& Schoeni, R. (2006), 'The Panel Study of Income Dynamics : Overview and Summary of Scientific Contributions After Nearly 40 Years', Survey Research Center Institute for Social Research - University of Michigan - Technical Series Paper 06-01.

Meyer, B. (1990), 'Unemployment Insurance and Unemployment Spells', Econometrica 58(4), 757-782.

Munch, J.-R., Rosholm, M. \& Svarer, M. (2006), 'Are Home Owners Really More Unemployed?', The Economic Journal 116(514), 991-1013.

Murray, P. (2007), Real Estate Conveyancing in 5 European Union Member States : A Comparative Study, http ://www.cnue-nouvelles.be/en/000/actualites/murray-report-final.pdf.

Oswald, A. (1996), 'A conjecture on the explanation for high unemployment in the industrialised nations : part 1', University of Warwick Economic Research Papers $4 \mathbf{7 5 .}$

Oswald, A. (1998), 'The missing piece of unemployment puzzle', CEPR Workshop on Unemployment Dynamics .

Oswald, A. (1999), The Housing Market and Europe's Unemployment : A Non-Technical Paper, unpublised paper, www2.warwick.ac.uk/fac/soc/ economics/staff/faculty/oswald/homesnt.pdf.

Peto, R. \& Peto, J. (1972), 'Asymptotically efficient rank invariant test procedures (with discussion)', Journal of the Royal Statistical Society 135, 185-206.

Prentice, R. (1978), 'Linear rank tests with right censored data', Biomatrika 65, 167-179.

Simpson, W. (1992), Urban Structure and the Labour Market : Worker Mobility, Commuting and Underemployment in Cities, Clarendon Press, Oxford.

van den Berg, G. (2001), Duration models : specification, identification and multiple durations, in J. Heckman \& E. Leamer, eds, 'Handbook of Econometrics', Amsterdam : North Holland, pp. 3381-3460. 
van Vuuren, A. (2009), The impact of Homeownership on Unemployment in the Netherlands, in C. van Ewijk \& M. van Leuvensteijn, eds, 'Homeownership \& the Labour Market in Europe', Oxford University Press, chapter 5, pp. 113-135.

Zenou, Y. (2009), Urban Labor Economics, Cambridge University Press. 